\title{
Protograph EXIT Analysis over Block Fading Channels with Application to Relays
}

\author{
Paola Pulini, Gianluigi Liva, and Marco Chiani
}

\begin{abstract}
An accurate extrinsic information transfer (EXIT) analysis is developed for protograph low-density parity-check (LDPC) codes over the block fading channel (BFC). The analysis is thus exploited for analyzing the behavior of distributed protograph LDPC ensembles over block fading relay channels. A novel protograph-based construction of low-density parity-check (LDPC) codes for the relay channel is proposed, which provides an enhanced unequal error protection (named unequal diversity, UD) property. The focus is on quasi-static fading channels and on the high-code-rate $(R>1 / 2)$ regimes, for which (according to the Singleton bound) no full diversity can be achieved. In the proposed construction, some nodes (and the corresponding codeword fragments) associated with the code graph enjoy the diversity provided by the relay, whereas the remaining nodes do not experience any diversity. The proposed approach can be thus tailored to transmit information blocks with different priority levels.
\end{abstract}

\section{INTRODUCTION}

Renewed interest in low-density parity-check (LDPC) codes [1] stems from the application of coding techniques to wireless block-fading and relay channels [2]-[10].

In this paper, we introduce a simple yet accurate extrinsic information transfer (EXIT) analysis for protograph-based LDPC codes [11], [12] over the block fading channel (BFC). The proposed analysis is an extension of the protograph extrinsic information transfer (PEXIT) analysis of [13]. The proposed approach simplifies the density evolution (DE) analysis of [7] for protograph LDPC ensembles, allowing a fast and accurate evaluation of the outage behavior of protograph LDPC ensembles. Additionally, it allows discriminating the behavior of the different protograph variable nodes.

We adopt the developed tools to design and analyze some distributed protograph ensembles in the framework of a singlerelay communication system operating in quasi-static Rayleigh fading. High-rate $(R>1 / 2)$ distributed LDPC coded schemes are introduced. The peculiarity of the proposed schemes resides in the so-called unequal diversity (UD) achieved by the different codeword fragments. More specifically, we design distributed LDPC codes which allow achieving diversity- 2 for some codeword fragments, whereas the remaining codeword fragments do not enjoy diversity. The proposed scheme thus trades diversity with coding rate (in fact, diversity- 2 cannot be achieved by any scheme with $R>1 / 2$ over a 2-levels BFC)

Gianluigi Liva and Paola Pulini are with Institute of Communication and Navigation of the Deutsches Zentrum fur Luft- und Raumfahrt (DLR), 82234 Wessling, Germany (e-mail: \{Paola.Pulini, Gianluigi.Liva\}@dlr.de).

Marco Chiani is with DEIS/WiLAB, University of Bologna, 47023 Cesena (FC), Italy (e-mail: marco.chiani@unibo.it). by re-encoding at the relay a subset of the information bits encoded at the source. The proposed scheme is hence of interest for any communication system for which the unequal error protection (UEP) concept is relevant. Note that the PEXIT analysis has been previously adopted to design bilayer [5] protographs for the relay channel [10]. Nevertheless, in [10] the authors derive via PEXIT analysis the iterative decoding threshold over additive white Gaussian noise (AWGN) channel only for the designed protographs, focusing on the different point-to-point links and hence without any modification of the original analysis of [12], [13]. We extend the definition of outage regions of a code ensemble [4] to distributed protograph ensembles and to protograph variable nodes. An example of a distributed protograph ensemble is provided, targeting the high coding rate regime $(R>1 / 2)$. Via PEXIT analysis, we develop simple tight bounds on the fragment error probability (FEP) associated with different codeword fragments. Numerical results confirm the accuracy of the proposed analysis.

The rest of the paper is organized as follows. The PEXIT ensemble analysis is described in Section II. In Section III we review the considered relay transmission scheme and unequal diversity protographs. The PEXIT analysis is exploited to characterize the relay scheme performance in Section IV. Conclusions follow in Section V.

\section{Protograph EXIT ANAlysis OVER Block FADING CHANNELS}

A protograph [11], [12], [14] is a Tanner graph [15] with a relatively small number of nodes. A protograph $\mathcal{G}=$ $(\mathscr{V}, \mathscr{C}, \mathscr{E})$ consists of a set of $N$ variable nodes $\mathscr{V}$, a set of $M$ check nodes $\mathscr{C}$, and a set of edges $\mathscr{E}$. Each edge $e_{i, j} \in \mathscr{E}$ connects a variable node $V_{j} \in \mathscr{V}$ to a check node $C_{i} \in \mathscr{C}$. Multiple parallel edges are permitted. A larger graph can be obtained by a copy-and-permute procedure: the protograph is copied $q$ times, and then the edges of the individual replicas are permuted among the $q$ replicas. The derived graph will consist of $n=N q$ variable nodes and $m=M q$ check nodes. A protograph can be described by a base matrix $\mathbf{B}$ of size $M \times N$. The element $b_{i, j}$ of $\mathbf{B}$ represents the number of edges connecting the variable node $V_{j}$ to the check node $C_{i}$.

In the following, we will consider protograph ensembles over non-ergodic BFCs. More specifically, we will consider a BFC with $N$ fading levels, one for each protograph variable node (VN). Roughly speaking, the protograph VNs will see independent channels with different signal-to-noise ratios (SNRs), distributed as

$$
f_{\gamma}(\gamma)=\frac{1}{\bar{\gamma}} e^{-\frac{\gamma}{\bar{\gamma}}},
$$


for $\gamma \geq 0$, while $f_{\gamma}(\gamma)=0$ for $\gamma<0$. We will first modify the PEXIT of [12], [13] to handle the BFC setting. Then, outage regions will be defined and the asymptotic ensemble error probability will be analyzed.

\section{A. Protograph EXIT Analysis}

We focus on the case in which each variable node is allowed to see a different channel. Define $I_{c h}^{(j)}$ the channel mutual information (MI) at the input of the $j$-th node. Define:

$I_{E v}(i, j)$ : the MI between the message sent by $V_{j}$ to $C_{i}$ and the associated codeword bit, on one of the $b_{i, j}$ edges connecting $V_{j}$ to $C_{i} \cdot\left(I_{E v}(i, j)=0\right.$ if $\left.b_{i, j}=0\right)$

$I_{E c}(i, j)$ : the MI between the message sent by $C_{i}$ to $V_{j}$ and the associated codeword bit, on one of the $b_{i, j}$ edges connecting $C_{i}$ to $V_{j} \cdot\left(I_{E c}(i, j)=0\right.$ if $\left.b_{i, j}=0\right)$

$I_{A P P}(j)$ : the MI between the a-posteriori probability (APP) log-likelihood ratio (LLR) (modeled as a random variable)

$$
L^{(j)}=\ln \frac{\operatorname{Pr}\left(x_{j}=+1 \mid \mathbf{y}\right)}{\operatorname{Pr}\left(x_{j}=-1 \mid \mathbf{y}\right)},
$$

evaluated by $V_{j}$ and the associated codeword bit $x_{j}$.

Suppose a variable node $V_{j}$ connected to $C_{i}$ (i.e., $b_{i, j} \neq 0$ ). The mutual information between the message sent by $V_{j}$ to $C_{i}$ and the associated codeword bit, over one of the $b_{i, j}$ edges connecting the nodes, will be a function of

- the channel MI $I_{c h}^{(j)}$;

- the a priori MI received by $V_{j}$ on each of the $b_{s, j}$ edges connecting $V_{j}$ to $C_{s}$, with $s \neq i$, weighted by $b_{s, j}$;

- the a priori MI received by $V_{j}$ on each of the $\left(b_{i, j}-1\right)$ edges connecting $V_{j}$ to $C_{i}$, weighted by $\left(b_{i, j}-1\right)$.

The MI between the message sent by $C_{i}$ to $V_{j}$ and codeword bit associated to $V_{j}$, over one of the $b_{i, j}$ edges connecting the nodes, will be a function of

- the a priori MI received by $C_{i}$ on each of the $b_{i, s}$ edges connecting $C_{i}$ to $V_{s}$, with $s \neq i$, weighted by $b_{i, s}$;

- the a priori MI received by $C_{i}$ on each of the $\left(b_{i, j}-1\right)$ edges connecting $C_{i}$ to $V_{j}$, weighted by $\left(b_{i, j}-1\right)$.

The MI between the a posteriori probability log-likelihood ratio $L^{(j)}$ evaluated by $V_{j}$ and the associated codeword bit will depend on the MI of all the incoming messages of $V_{j}$.

Once the above-mentioned functions have been obtained for a generic channel, and once the channel nuisance parameter has been fixed, the MI evolution along the edges of the graph can be computed iteratively, recalling that the MI on an edge connecting $V_{j}$ and $C_{i}$, at the output of the variable node, is the a priori MI for $C_{i}$, i.e., $I_{E v}(i, j)=I_{A c}(i, j)$. Similarly, $I_{E c}(i, j)=I_{A v}(i, j)$. At each iteration a set of $I_{A P P}(j)$, with $i=0 \ldots N-1$, is produced.

The EXIT functions for variable/check nodes on the AWGN channel have been introduced in [16]. We denote with $J(\sigma)$ the MI between a binary random variable $X$, with $\operatorname{Pr}(X=$ $+\mu)=1 / 2$ and $\operatorname{Pr}(X=-\mu)=1 / 2$, and a continuous Gaussian-distributed random variable $Y$ with mean $X$ and variance $\sigma^{2}=2 \mu$ (symmetry condition). $J(\sigma)$ represents the capacity of a binary-input additive Gaussian noise channel, and it is given by [17]

$$
J(\sigma)=1-\int_{-\infty}^{+\infty} \frac{1}{\sqrt{2 \pi \sigma^{2}}} e^{-\frac{\left(y-\sigma^{2} / 2\right)^{2}}{2 \sigma^{2}}} \cdot \log _{2}\left(1+e^{-y}\right) d y
$$

Simple approximations of the functions $J(\cdot)$ and $J^{-1}(\cdot)$ can be found in [16]. The PEXIT analysis proceeds as follows.

\section{1) Initialization}

Initialize $I_{c h}^{(j)}=J\left(\sigma_{c h, j}\right), \forall j=0 \ldots N-1$, with

$$
\sigma_{c h, j}^{2}=8 \gamma^{(j)}
$$

where $\gamma^{(j)}$ represents the signal-to-noise ratio associated to the channel input to the $j$-th variable node. We define the channel profile as the vector of instantaneous SNRs $\gamma=\left[\gamma^{(0)}, \gamma^{(1)}, \ldots, \gamma^{(N-1)}\right]$.

2) Variable to check update

For $j=0, \ldots, N-1$ and $i=0, \ldots, M-1$, if $b_{i, j} \neq 0$,

$$
\begin{aligned}
& I_{E v}(i, j) \approx \\
& \left.\frac{J\left(\sqrt{\sum_{s \neq i} b_{s, j}\left[J^{-1}\left(I_{A v}(s, j)\right)\right]^{2}+}\right.}{+\left(b_{i, j}-1\right)\left[J^{-1}\left(I_{A v}(i, j)\right)\right]^{2}+\left[J^{-1}\left(I_{c h}^{(j)}\right)\right]^{2}}\right) .
\end{aligned}
$$

If $b_{i, j}=0, I_{E v}(i, j)=0$.

3) For $j=0, \ldots, N-1$ and $i=0, \ldots, M-1$, set $I_{A c}(i, j)=I_{E v}(i, j)$.

4) Check to variable update

For $j=0, \ldots, N-1$ and $i=0, \ldots, M-1$, if $b_{i, j} \neq 0$,

$$
\begin{aligned}
& I_{E c}(i, j) \approx \\
& 1-J\left(\sqrt{\sum_{s \neq j} b_{i, s}\left[J^{-1}\left(1-I_{A c}(i, s)\right)\right]^{2}+}\right. \\
& \left.\overline{+\left(b_{i, j}-1\right)\left[J^{-1}\left(1-I_{A c}(i, j)\right)\right]^{2}}\right) \text {. }
\end{aligned}
$$

If $b_{i, j}=0, I_{E c}(i, j)=0$.

5) For $j=0, \ldots, N-1$ and $i=0, \ldots, M-1$, set $I_{A v}(i, j)=I_{E c}(i, j)$.

6) APP-LLR mutual information evaluation For $j=0, \ldots, N-1$,

$$
\begin{aligned}
& I_{A P P}(j) \approx \\
& J\left(\sqrt{\sum_{s} b_{s, j}\left[J^{-1}\left(I_{A v}(i, j)\right)\right]^{2}+\left[J^{-1}\left(I_{c h}^{(j)}\right)\right]^{2}}\right) .
\end{aligned}
$$

The steps 2-6 are iterated until $I_{A P P}(j)=1, \forall j$, or a maximum number of iterations $\left(I_{\max }\right)$ is reached. For a given channel profile $\gamma$ the convergence of a VN $V_{j}$ APP to $I_{A P P}(j)=1$ means that, for the codeword bits associated with the $j$-th VN type, a vanishing error probability is achieved in the asymptotic setting. 


\section{B. Outage Regions and Asymptotic Error Probability}

We shall adapt next the definition outage region for a code ensemble introduced in [4] to protograph ensembles. More specifically, we will introduce the concept of outage regions for the protograph nodes.

Definition 1 (Convergence region of a protograph VN). We identify by

$$
\mathcal{D}^{(j)}=\left\{\boldsymbol{\gamma} \in \mathbb{R}_{+}^{N} \mid I_{A P P}(j)=1, I_{\text {max }} \rightarrow \infty\right\}
$$

as the $N$-dimensional convergence region of the $V N V_{j}$.

Definition 2 (Outage region of a protograph VN). We identify by

$$
\overline{\mathcal{D}}^{(j)}=\left\{\gamma \in \mathbb{R}_{+}^{N} \mid I_{A P P}(j)<1, I_{\max } \rightarrow \infty\right\}
$$

the $N$-dimensional outage region of the $V N V_{j}$.

Definition 3 (Convergence region of a protograph $\mathcal{G}$ ). We identify by

$$
\mathcal{D}_{\mathcal{G}}=\left\{\boldsymbol{\gamma} \in \mathbb{R}_{+}^{N} \mid I_{A P P}(j)=1, \forall j, I_{\max } \rightarrow \infty\right\}
$$

the $N$-dimensional convergence region of $\mathcal{G}$.

Definition 4 (Outage region of a protograph $\mathcal{G}$ ). We identify by

$$
\overline{\mathcal{D}}_{\mathcal{G}}=\left\{\boldsymbol{\gamma} \in \mathbb{R}_{+}^{N} \mid \exists j \text { s.t. } I_{A P P}(j)<1, I_{\text {max }} \rightarrow \infty\right\}
$$

the $N$-dimensional outage region of $\mathcal{G}$.

Under actual density evolution, the average ensemble block error probability can be lower-bounded as [7] ${ }^{1}$

$$
P_{B} \geq \int_{\overline{\mathcal{D}}_{\mathcal{G}}} \prod_{i=0}^{N-1} f_{\gamma}\left(\gamma^{(i)}\right) d \gamma^{(i)}
$$

while the average ensemble bit error probability is

$$
\begin{aligned}
P_{b} & =\mathbb{E}_{\boldsymbol{\gamma}}\left[P_{b}(\gamma)\right] \\
& =\int_{0}^{\infty} \int_{0}^{\infty} \cdots \int_{0}^{\infty} P_{b}(\gamma) \prod_{i=0}^{N-1} f_{\gamma}\left(\gamma^{(i)}\right) d \gamma^{(i)}
\end{aligned}
$$

where $P_{b}(\gamma)$ is the bit error probability averaged over the different protograph VNs for a given channel profile $\gamma$,

$$
P_{b}(\gamma)=\frac{1}{N} \sum_{i=0}^{N-1} P_{b}^{(i)}(\gamma)
$$

The bit error probability associated with the $i$-th $\mathrm{VN}$ is obtained as

$$
P_{b}^{(i)}(\gamma) \approx \frac{1}{2} \operatorname{erfc}\left(\frac{1}{2 \sqrt{2}} J^{-1}\left(I_{A P P}(i)\right)\right) .
$$

\footnotetext{
${ }^{1}$ The convergence to a vanishing bit error probability does not represent always a sufficient condition for the convergence to a vanishing block error probability [18], [19]. Nevertheless, often the right-hand side of (3) can be confidently used to estimate the block error probability of finite length LDPC codes [7]
}

Since $P_{b}^{(i)}$ vanishes outside $\overline{\mathcal{D}}^{(i)}$, we have that

$$
P_{b}=\frac{1}{N} \sum_{i=0}^{N-1} \int_{\overline{\mathcal{D}}^{(i)}} P_{b}^{(i)}(\gamma) \prod_{j=0}^{N-1} f_{\gamma}\left(\gamma^{(j)}\right) d \gamma^{(j)}
$$

We remark that (3)-(6) hold in an approximate sense due to the Gaussian approximation which is inherent to the EXIT analysis. Thus, all the bounds and equalities based on EXIT analysis have to be considered as approximations.

Definition 5 (Diversity of a protograph). The diversity achieved by a protograph is finally defined as [20]

$$
d=\lim _{\bar{\gamma} \rightarrow \infty}-\frac{\log P_{b}}{\log \bar{\gamma}}
$$

Definition 6 (Diversity of a protograph VN). Extending the definition above, the diversity of the $i$-th $V N$ is defined as

$$
d_{i}=\lim _{\bar{\gamma} \rightarrow \infty}-\frac{\log P_{b}^{(i)}}{\log \bar{\gamma}} .
$$

The UEP property of the protographs presented in this paper resides in assigning the high-priority fragments to the nodes with largest diversity. In the following, a cooperative transmission scheme is introduced, whose performance can be conveniently characterized by means of the proposed PEXIT analysis.

\section{An UneQual DiVersity CoOperative TRANSMISSION SCHEME}

Following Figures 1(a) and 1(b), a rate- $R_{S}$ code $\mathcal{C}_{S}$ is employed at the source to encode a length- $k$ message vector $\mathbf{u}$ which can be split into two sub-blocks (fragments, in the following) as $\mathbf{u}=\left[\mathbf{u}_{h} \mid \mathbf{u}_{l}\right]$, where $\mathbf{u}_{h}$ represents the $k_{h}$ bits long high-priority fragment and $\mathbf{u}_{l}$ represents the $k_{l}$ bits long low-priority fragment. The length- $n_{S}$ coded block is given by $\mathbf{c}_{S}=\left[\mathbf{u} \mid \mathbf{p}_{S}\right]$, where $\mathbf{p}_{S}$ is the $m_{S}=n_{S}-k$ bits parity vector. The coded block is then BPSK modulated, resulting in the modulated sequence $\mathbf{x}_{S}$ (with $\mathbf{x}_{S} \in\{-1,+1\}^{n_{S}}$ ), and transmitted over the first time slot. A corrupted version of $\mathbf{x}_{S}$ is received at the destination as

$$
\mathbf{y}_{S}=\alpha_{S} \mathbf{x}_{S}+\mathbf{n}_{S},
$$

where $\alpha_{S}$ follows a Rayleigh distribution with $\mathbb{E}\left[\alpha_{S}^{2}\right]=1$, and where the elements $\mathbf{n}_{S}$ are i.i.d. Gaussian random variables with variance $\sigma_{n}^{2}=N_{0} / 2$. The instantaneous SNR is given by $\gamma_{S}=\alpha_{S}^{2} / 2 \sigma_{n}^{2}$.

The same sequence $\mathbf{x}_{S}$ is received at the relay. We will make use of a simplified assumption, i.e. we will consider the link between source and relay as reliable [8]. The assumption is justified by considering the case where the relay is selected among a set of candidate nodes in such a way the SNR on the S-R link is large enough to achieve negligible error 


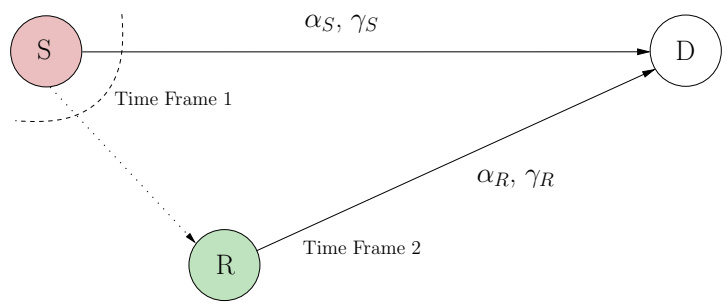

(a) Relay transmission scheme.

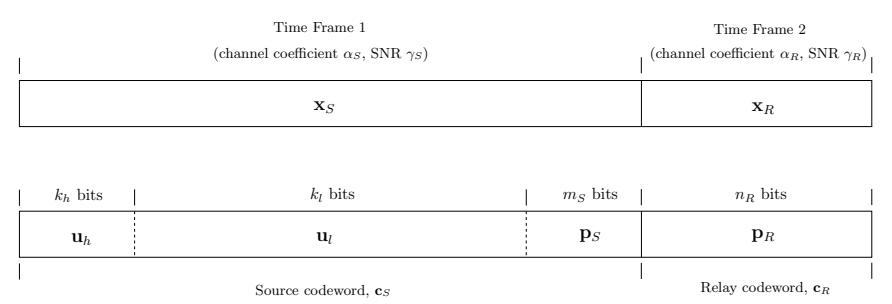

(b) Frame structure

Fig. 1. Overview of the transmission scheme and of the frame structure.

probabilities after decoding. ${ }^{2}$ In this sense, the introduction of coding at the source (through $\mathcal{C}_{S}$ ) is essential to permit the selection of the relaying partner in a suitably-large area in the neighborhood of the source terminal. Note moreover that under the simplified condition the challenges in the code design remain substantially unchanged [8]. After decoding of the block $\mathbf{u}$, the relay extracts the high-priority fragment $\mathbf{u}_{h}$ and performs encoding with a rate- $R_{R}$ non-systematic code $\mathcal{C}_{R}$, producing the code block $\mathbf{c}_{R}=\left[\mathbf{p}_{R}\right]$, where $\mathbf{p}_{R}$ is a $n_{R}$ bits vector. The coded block is then BPSK modulated, resulting in the modulated sequence $\mathbf{x}_{R}$ (with $\mathbf{x}_{R} \in\{-1,+1\}^{n_{R}}$ ), and transmitted over the second time slot. A corrupted version of $\mathbf{x}_{R}$ is received at the destination as

$$
\mathbf{y}_{R}=\alpha_{R} \mathbf{x}_{R}+\mathbf{n}_{R},
$$

where $\alpha_{R}$ follows a Rayleigh distribution with $\mathbb{E}\left[\alpha_{R}^{2}\right]=1$, and where the elements $\mathbf{n}_{R}$ are i.i.d. Gaussian random variables with variance $\sigma_{n}^{2}=N_{0} / 2$. The instantaneous SNR is given by $\gamma_{R}=\alpha_{R}^{2} / 2 \sigma_{n}^{2}$.

At the receiver side, the joint observation $\mathbf{y}=\left[\mathbf{y}_{S} \mid \mathbf{y}_{R}\right]$ is formed and it is input to the decoder for the joint code $\mathcal{C}$ whose task if to retrieve the code word $\mathbf{x}=\left[\mathbf{x}_{S} \mid \mathbf{x}_{R}\right]$, where the two blocks $\mathbf{x}_{S}, \mathbf{x}_{R}$ experience to two independent fading levels $\alpha_{S}, \alpha_{R}$.

\section{A. Parity-Check Matrix Structure and Diversity}

The overall $\mathcal{C}$ code parity-check matrix will have form

$$
\mathbf{H}=\left[\begin{array}{c|c|c}
\multicolumn{2}{|c|}{\mathbf{H}_{S}} & \mathbf{0} \\
\hline \mathbf{M} & \mathbf{0} & \mathbf{P}
\end{array}\right],
$$

where the $\mathbf{H}_{S}$ is the $m_{S} \times n_{S}$ parity-check matrix of the code $\mathcal{C}_{S}$ and where $\mathcal{C}_{R}$ has extended $n_{R} \times\left(k_{h}+n_{R}\right)$ matrix in the form $^{3} \mathbf{H}_{R}^{e}=[\mathbf{M} \mid \mathbf{P}]$ where $\mathbf{M}$ is a $n_{R} \times k_{h}$ associated with

\footnotetext{
${ }^{2}$ This assumption is realistic in many cases. As an example, the aeronautical (in-airport) communications represent an ideal candidate application for the technique proposed in this paper. The airport communication channel [21] confirms the hypothesis considered in our analysis (i.e., slow Rayleigh fading), especially for scenarios where the vehicles on the airport surface are moving at moderate speeds or are in parking position. Indeed, the relay terminal (which is an aircraft) may be easily chosen in order to guarantee a very high SNR between source and relay, since often there is the possibility of selecting a relay that are standing close to the transmitting aircraft. Furthermore, the traffic profiles considered for aeronautical applications, which are defined by [22], includes three different classes of messages with different requirements. Hence, the use of a method which implicity provides different protection for different information constitutes a valuable feature.

${ }^{3}$ We have that $\mathbf{H}_{R}^{e}\left[\mathbf{u}_{h}, \mathbf{p}_{R}\right]^{T}=\mathbf{0}^{T}$, i.e. $\mathbf{H}_{R}^{e}$ is the parity-check matrix of the augmented code obtained by concatenating $\mathbf{u}_{h}$ and $\mathbf{p}_{R}$.
}

the information bits in $\mathbf{u}_{h}$ (which are not transmitted by the relay), and $\mathbf{P}$ is the $n_{R} \times n_{R}$ matrix associated with the parity vector $\mathbf{p}_{R}$. Note that the final code length is $n=n_{S}+n_{R}$, and the overall code rate is $R=k /\left(n_{S}+n_{R}\right)$.

Among the possible decoding strategies, two possibilities deserve particular interest. A first (optimum) approach deals with joint decoding of the two codes $\mathcal{C}_{S}, \mathcal{C}_{R}$ composing the distributed code $\mathcal{C}$. A low-complexity alternative is represented by a scheme which decodes $\mathcal{C}_{S}$ and $\mathcal{C}_{R}$ separately, ${ }^{4}$ and depending on the decoders outcome delivers either $u_{h}$ or $u_{l}$, or both (or none of them, if none of the decoders is successful). ${ }^{5}$ Note that in both cases the high-priority fragment achieves diversity of order 2 if $\operatorname{rank} \mathbf{M}=k_{h}$, i.e. if each non-zero codeword of the augmented code having parity-check matrix $\mathbf{H}_{R}^{e}$ has its support spread over $\mathbf{u}_{h}$ and $\mathbf{p}_{R}$.

This behavior can be achieved by a distributed LDPC scheme under joint iterative decoding of $\mathcal{C}$. Consider the parity-check matrix $\mathbf{H}_{S}$ of the code $\mathcal{C}_{S}$, where $\mathcal{C}_{S}$ is a lowdensity parity-check code. Moreover, we assume $\mathcal{C}_{R}$ to be a non-systematic low-density parity-check code with extended low-density parity-check matrix in the form $\mathbf{H}_{R}^{e}=[\mathbf{M} \mid \mathbf{P}]$. The overall $\mathcal{C}$ code parity-check matrix will be low density. Decoding can thus be performed on the Tanner graph associated with $\mathbf{H}$.

\section{B. Unequal Diversity Protographs}

The code structure presented above can be suitably realized by means of protograph LDPC code constructions. We introduce the concepts of source protograph $\mathcal{G}_{S}$, by which we denote the protograph for the (high-rate) code employed at the source, and of relay protograph $\mathcal{G}_{R}$, which refers to the code employed at the relay. The two protographs are connected via the VNs associated with the bits of $\mathbf{u}_{h}$. The union of the two protographs gives rise to the distributed protograph $\mathcal{G}$, which defines the distributed LDPC code ensemble employed by the cooperative scheme. The scheme can be seen as a distributed parallel concatenation of two block codes [23] where the concatenation involves only a fraction of the information bits.

Example 1 - Rate 7/10 protograph. An first protograph example is depicted in Figure 2. The protograph relates to a rate

\footnotetext{
${ }^{4}$ Alternatively, one may consider forwarding the decision of the decoder associated with $\mathcal{C}_{R}$ (if successful) to the decoder associated with $\mathcal{C}_{S}$. This has the effect of shortening $\mathcal{C}_{S}$ thus facilitating the recovery of $u_{l}$

${ }^{5}$ We assume that an error detection mechanism provides a validation of the decoders output.
} 
$R=7 / 10$ ensemble and consists of a parallel concatenation of a rate $R_{S}=7 / 8$ irregular repeat accumulate (IRA) protograph $\mathcal{G}_{S}$ with base matrix

$$
\mathbf{B}_{S}=\left[\begin{array}{llllllll}
4 & 4 & 4 & 4 & 4 & 4 & 4 & 2
\end{array}\right]
$$

and a rate $R_{R}=1 / 2$ non-systematic repeat accumulate (RA) protograph $\mathcal{G}_{R}$ with base matrix

$$
\mathbf{B}_{R}=\left[\begin{array}{lll}
1 & 1 & 1 \\
1 & 1 & 1
\end{array}\right]
$$

The overall distributed protograph $\mathcal{G}$ possesses a base matrix

$$
\mathbf{B}=\left[\begin{array}{llllllllll}
4 & 4 & 4 & 4 & 4 & 4 & 4 & 2 & 0 & 0 \\
1 & 0 & 0 & 0 & 0 & 0 & 0 & 0 & 1 & 1 \\
1 & 0 & 0 & 0 & 0 & 0 & 0 & 0 & 1 & 1
\end{array}\right]
$$

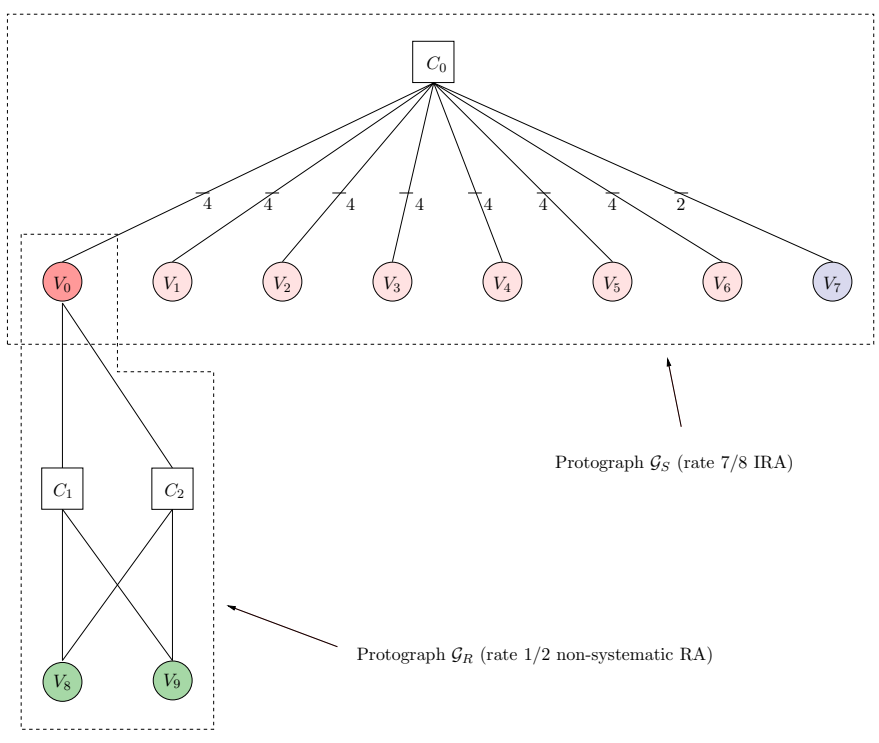

Fig. 2. Protograph from the Example 1. The IRA code is adopted at the source, whereas the RA is used for encoding the high-priority fragment at the relay. The first column of $\mathbf{B}_{R}$ is hence associated with the high-priority fragment (VN $V_{0}$ in the protograph), which is not transmitted by the relay.

\section{ANAlysis FOR THE RELAY CHANNEL}

The analysis presented in Section II can be easily adapted to the case of the relay channel with quasi-static fading. More specifically, we shall set to $\gamma^{(j)}=\gamma_{S}$ for all the protograph VNs associated with fragments transmitted over the sourcedestination channel. For the protograph VNs associated with fragments transmitted over the relay-destination channel we shall set $\gamma^{(j)}=\gamma_{R}$. We denote by $\mathcal{V}_{h}$ as the set of protograph VNs associated with high-priority fragments (thus, having the largest diversity), and by $\mathcal{V}_{l}$ as the set of protograph VNs associated with low-priority fragments. The convergence/outage regions of the variable nodes in $\mathcal{V}_{h}, \mathcal{V}_{l}$ depend on $\gamma_{S}$ and $\gamma_{R}$ only. Thus, they can be conveniently represented on the 2-dimensional $\gamma_{S} \times \gamma_{R}$ plane.

Definition 7 (Convergence region of high-priority fragments for a protograph $\mathcal{G})$. We identify by

$$
\mathcal{D}_{h}=\left\{\gamma_{S}, \gamma_{R} \mid I_{A P P}(j)=1, \forall V_{j} \in \mathcal{V}_{h}, I_{\max } \rightarrow \infty\right\}
$$

the 2-dimensional convergence region of the high-priority fragments for the $\mathcal{G}$.

Definition 8 (Convergence region of low-priority fragments for a protograph $\mathcal{G})$. We identify by

$$
\mathcal{D}_{l}=\left\{\gamma_{S}, \gamma_{R} \mid I_{A P P}(j)=1, \forall V_{j} \in \mathcal{V}_{l}, I_{\max } \rightarrow \infty\right\}
$$

the 2-dimensional convergence region of the low-priority fragments for the $\mathcal{G}$.

The corresponding complementary non-convergence (outage) regions are given by $\overline{\mathcal{D}}_{h}$ and $\overline{\mathcal{D}}_{l}$.

The asymptotic (in the block length) FEPs associated with high and low-priority fragments can be bounded by

$$
\begin{aligned}
& P_{F}^{(h)} \geq \int_{\overline{\mathcal{D}}_{h}} f_{\gamma}\left(\gamma_{S}\right) f_{\gamma}\left(\gamma_{R}\right) d \gamma_{S} d \gamma_{R}, \\
& P_{F}^{(l)} \geq \int_{\overline{\mathcal{D}}_{l}} f_{\gamma}\left(\gamma_{S}\right) f_{\gamma}\left(\gamma_{R}\right) d \gamma_{S} d \gamma_{R}
\end{aligned}
$$

Example 1 - Rate 7/10 protograph. Figure 3(a) depicts the convergence regions for the high- and the low-priority fragments associated with the protograph of Figure 2 on the $\gamma_{S} \times \gamma_{R}$ plane (linear scale). A few observations follow.

A. When fixing $\gamma_{S}=0$, the border of the convergence region of the high priority fragment is at $\gamma_{R}=\gamma_{R}^{*} \simeq 1.03(0.142$ $\mathrm{dB}$, point $\mathrm{A}$ in the chart), which is the decoding threshold over the AWGN channel for the protograph associated with the base matrix $\mathbf{B}_{R}$.

B. When fixing $\gamma_{R}=0$, the border of the two convergence regions is given by $\gamma_{S}=\gamma_{S}^{*} \simeq 1.86(2.709 \mathrm{~dB}$, point $\mathrm{B}$ in the chart), which is the decoding threshold over the AWGN channel for the protograph associated with the base matrix $\mathbf{B}_{S}$.

C. For increasing $\gamma_{R}$, the border of the convergence region of the low priority fragments moves leftwards, and for sufficiently large $\gamma_{R}$ it achieves the value $\gamma_{S}=\gamma_{S}^{\prime} \simeq$ 1.74 (2.42 dB, point $\mathrm{C}$ in the chart), which is the decoding threshold over the AWGN channel for the protograph

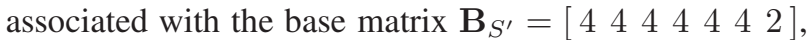
i.e. with a shortened version of the base matrix $\mathbf{B}_{S}$, where the first column (VN $V_{0}$ in the protograph) is removed due to the infinite reliability associated with the extrinsic information provided by the RA adopted at the relay (operated in its convergence region).

The evaluation of Eqs. (7) and (8) is provided in Figure 3(b) vs. the average SNR $\bar{\gamma}$, together with simulation results for the FER of a $n=2560$ code obtained by a 256-fold expansion of the protograph of Figure 2. The expansion has been performed in 2 steps via a circulant version of the progressive edge growth (PEG) algorithm [24], leading to structured (quasicyclic) IRA [25] and RA [26] codes at the source and at the relay respectively. The accuracy of the analysis is evident down to the simulated FERs $\left(10^{-5}\right)$, and confirms the different levels of diversity attained by the different node types. 


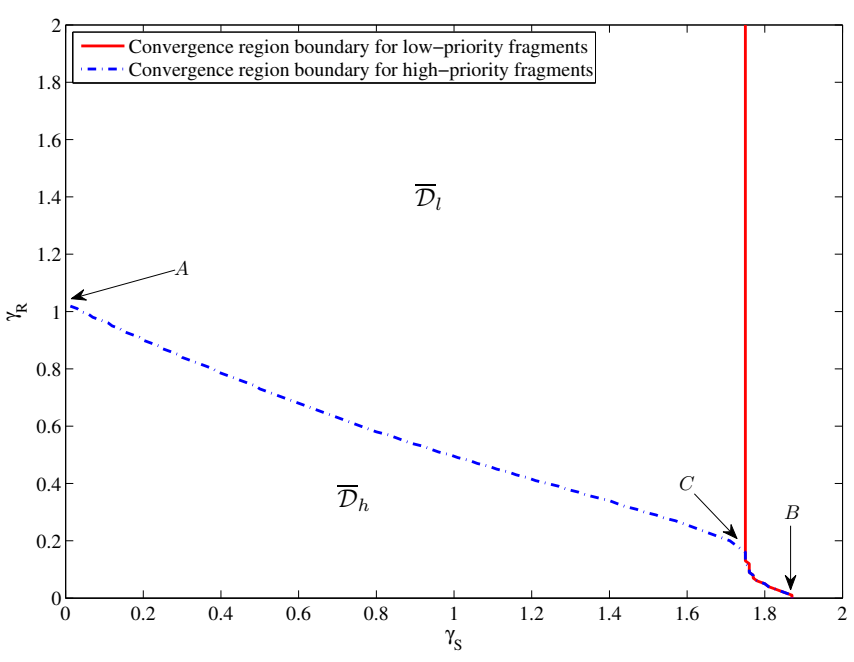

(a) Outage regions for the high- and the low-priority fragments.

Fig. 3. Outage regions and FEP $\left(P_{F}\right)$ for the protograph of Example 1.

\section{CONCLUSION}

In this paper, an accurate PEXIT analysis for the BFC has been introduced. The proposed analysis has been exploited to analyze the behavior of distributed protograph LDPC ensembles over the quasi-static relay fading channel. Protograph ensemble exhibiting the UD feature has been proposed, which are obtained via simple (parallel) concatenation of IRA/RA protographs. The proposed schemes allow combining coding efficiency (through high coding rates) with diversity for the high-priority parts of the source messages.

\section{REFERENCES}

[1] R. Gallager, Low-Density Parity-Check Codes. Cambridge, MA: M.I.T. Press, 1963.

[2] M. Chiani, A. Conti, and A. Ventura, "Evaluation of low-density parity-check codes over block fading channels," in IEEE International Conference on Communications, New Orleans, LA, Jun. 2000.

[3] E. Biglieri, Coding for wireless channels. New York: Springer-Verlag, 2005.

[4] J. Boutros, A. G. i Fàbregas, and E. Calvanese, "Analysis of coding on non-ergodic block fading channels," in Proc. 42th Annu. Allerton Conf. on Communication, Control, and Computing, Allerton, Illinois, Oct. 2005.

[5] P. Razaghi and W. Yu, "Bilayer Low-Density Parity-Check Codes for Decode-and-Forward in Relay Channels," IEEE Trans. Inf. Theory, vol. 53, no. 10, pp. 3723-3739, Oct. 2007.

[6] J. Boutros, "Diversity and coding gain evolution in graph codes," in Proc. Information Theory and Applications Workshop, UCSD, San Diego, CA, Feb. 2009.

[7] J. Boutros, A. Guillén i Fàbregas, E.Biglieri, and G. Zemor, "LowDensity Parity-Check Codes for Nonergodic Block-Fading Channels," IEEE Trans. Inf. Theory, vol. 56, no. 9, pp. 4286 - 4300, Sep. 2010.

[8] D. Duyck, D. Capirone, J. Boutros, and M. Moeneclaey, "Analysis and construction of full-diversity joint network-LDPC codes for cooperative communications," EURASIP Journal on Wireless Communications and Networking, pp. 1-16, 2010.

[9] D. Duyck, M. Moeneclaey, M. Azmi, Y. Jinhong, and J. Boutros, "Universal LDPC codes for cooperative communications," in Proc. 6th International Symposium on Turbo Codes and Iterative Information Processing, Brest, France, Sep. 2010.

[10] T. V. Nguyen, A. Nosratinia, and D. Divsalar, "Bilayer protograph codes for half-duplex relay channels," in Proc. IEEE International Symposium on Information Theory (ISIT), Austin, TX, Jun. 2010.

[11] J. Thorpe, "Low-Density Parity-Check (LDPC) Codes Constructed from Protographs," JPL INP, Tech. Rep., Aug. 2003, 42-154.

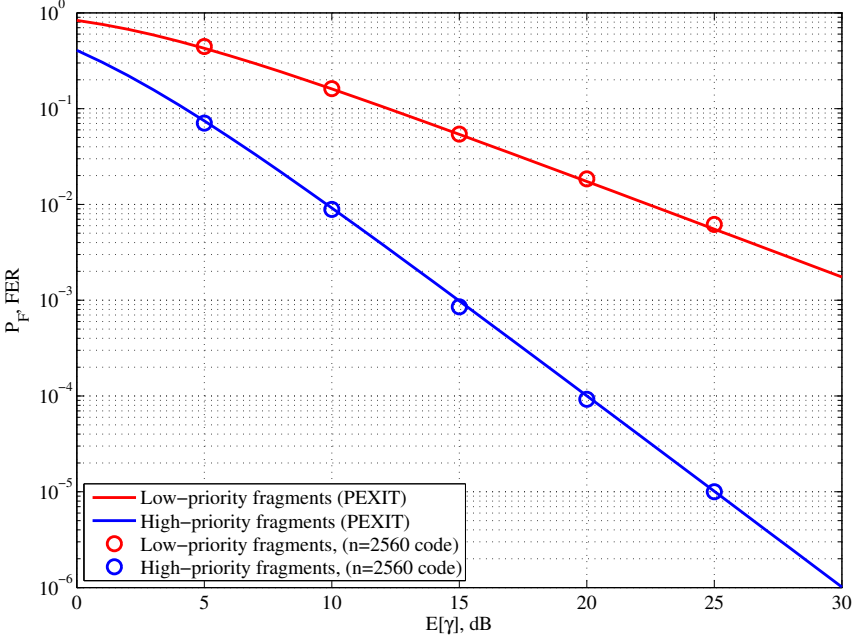

(b) FER performance vs. average SNR, compared with (7) and (8).
[12] G. Liva, S. Song, L. Lan, Y. Zhang, W. Ryan, and S. Lin, "Design of LDPC codes: A survey and new results," J. Commun. Softw. Syst., vol. 2, no. 3, pp. 191-211, Sep. 2006, invited paper.

[13] G. Liva and M. Chiani, "Protograph LDPC codes design based on EXIT analysis," in Proc. IEEE Globecomm, Washington, D.C., USA, Nov. 2007.

[14] W. E. Ryan and S. Lin, Channel Codes - Classical and Modern. Cambridge University Press, 2009.

[15] R. Tanner, "A recursive approach to low complexity codes," IEEE Trans. Inf. Theory, vol. 27, pp. 533-547, Sep. 1981.

[16] S. ten Brink, G. Kramer, and A. Ashikhmin, "Design of low-density parity-check codes for modulation and detection," IEEE Trans. Commun., vol. 52, pp. 670-678, Apr. 2004.

[17] S. ten Brink, "Convergence behavior of iteratively decoded parallel concatenated codes," IEEE Trans. Commun., vol. 49, pp. 1727-1737, Oct. 2001.

[18] M. Lentmaier and D.V. Truhachev and K.Sh. Zigangirov and D.J. Costello, "An analysis of the block error probability performance of iterative decoding," IEEE Trans. Inf. Theory, vol. 51, no. 11, pp. 3834 - 3855, Nov. 2005.

[19] H. Jin and T. Richardson, "Block Error Iterative Decoding Capacity for LDPC Codes," in Proc. of IEEE Int. Symp. on Information Theory, Sep. 2005, pp. 52-56.

[20] D. Tse and P. Viswanath, Fundamentals of Wireless Communication. Cambridge University Press, 2005.

[21] P. Pulini, "Forward Link Performance Analysis for the Future IEEE 802.16-based Airport Data-Link," in Proc. IEEE Int. Conf. on Communications, Cape Town, South Africa, May 2010.

[22] "Communications operating concept and requirements for the future radio system (cocr)," EUROCONTROL/ Federal Aviation Administration (FAA) - Future Communications Study Operational Concepts and Requirements Team, Tech. Rep. 2.0, 2007.

[23] M. Valenti and B. Zhao, "Distributed turbo codes: Towards the capacity of the relay channel," in Proc. IEEE Vehicular Tech. Conf. (VTC), Orlando, FL, Oct. 2003.

[24] X.-Y. Hu, E. Eleftheriou, and D. Arnold, "Regular and irregular progressive edge-growth Tanner graphs," IEEE Trans. Inf. Theory, vol. 51, no. 1, pp. 386-398, Jan. 2005.

[25] Y. Zhang and W. E. Ryan, "Structured IRA codes: Performance analysis and construction," IEEE Trans. Commun., vol. 55, no. 5, pp. 837-844, May 2007.

[26] R. Tanner, "On quasi-cyclic repeat-accumulate codes," in Proc. 37th Annu. Allerton Conf. on Communication, Control, and Computing, Monticello, Illinois, Sep. 1999. 